On wave-particle interaction in axisymmetric toroidal systems

This article has been downloaded from IOPscience. Please scroll down to see the full text article.

2011 Phys. Scr. 84015503

(http://iopscience.iop.org/1402-4896/84/1/015503)

View the table of contents for this issue, or go to the journal homepage for more

Download details:

IP Address: 129.16.198.136

The article was downloaded on 21/06/2011 at 09:59

Please note that terms and conditions apply. 


\title{
On wave-particle interaction in axisymmetric toroidal systems
}

\author{
R M Nyqvist ${ }^{1}$, M Lisak ${ }^{1}$ and $\mathrm{J}$ Zalesny $^{2}$ \\ ${ }^{1}$ Earth and Space Sciences, Chalmers University of Technology, SE-412 96 Göteborg, Sweden \\ ${ }^{2}$ Institute of Physics, West Pomeranian University of Technology, 70-310 Szczecin, Poland \\ E-mail: robert.nyqvist@chalmers.se
}

Received 16 May 2011

Accepted for publication 24 May 2011

Published 13 June 2011

Online at stacks.iop.org/PhysScr/84/015503

\begin{abstract}
A general formalism is developed to describe the interaction of charged particles with electromagnetic waves in terms of coupled finite difference mapping equations that incorporate tokamak topology. The approach is based on considering non-adiabatic changes in the constants of particle motion and it covers a range of wave-particle resonance frequencies, from the precessional to cyclotron frequencies of both passing and trapped ions. The concept of overlapping resonances is used to estimate the threshold for a single plane wave to cause stochastic particle motion. In the stochastic regime, the process is Markovian, and particle diffusion in three-dimensional phase space takes place. Estimations of diffusion coefficients are carried out in the two cases of waves interacting with passing and trapped ions by means of the cyclotron and bounce resonances, respectively, and previously known results are recovered in the proper limits.
\end{abstract}

PACS numbers: 52.20.Dq, 52.25.Gj, 52.55.Fa

(Some figures in this article are in colour only in the electronic version.)

\section{Introduction}

In general, wave-particle interaction involves multiple resonances [1-6], and the particle motion will qualitatively differ depending on whether these resonances overlap or not. The concept of overlapping resonances yields a physical explanation for the ability of a single wave to cause stochastic particle motion. The important point is that along its trajectory, a charged particle will experience electromagnetic oscillations not at the single frequency $\omega$ of the wave, but at many frequencies differing from $\omega$ by multiples of the bounce and cyclotron frequencies. The resulting complicated, stochastic particle motion is characterized by the destruction of one or more constants of motion, and the evolution of stochastically moving particles may be described as a diffusive process.

Overlap of resonances occurs in many problems associated with magnetically confined plasmas. In a nonuniform magnetic field, ions can move stochastically in the presence of a wave instability. Such problems have been studied for tokamaks [7] and for mirror machines [1, 2, 8]. The interaction between a wave and a charged particle is of practical interest in connection with radio frequency (RF) heating of tokamak plasmas, where the RF-waves may effect the confinement characteristics of the plasma. In particular, a potential problem associated with RF-heating in the ion cyclotron frequency range is the possibility of plasma confinement degradation due to ion cyclotron wave induced diffusion [9-11]. A theory of wave-particle interaction in the presence of RF-waves, with frequencies much lower than the cyclotron frequency of trapped particles, in tokamaks was presented in [12, 13]. Stochastic ion motion has also been found for the lower hybrid heating scheme in tokamaks [14-16]. Another interesting issue is connected with the conditions under which it is possible to apply quasi-linear theory to describe the heating processes.

The aim of the present paper is to develop a general formalism to describe wave-particle interaction in a tokamak. The approach is based on the concept of overlapping resonances and results in a set of finite difference mapping equations for the constants of particle motion. The main advantage of this approach is that the number of phase space variables is less than that which appears in the Hamiltonian formalism [1-6, 16, 17]. 
The paper is organized in the following manner. In section 2, some basic concepts of particle motion in tokamaks are discussed. In section 3 , the equations describing time evolution of the invariants of particle motion are derived and solved by means of the stationary phase method. Finally, in section 4 , a set of finite difference mapping equations for the invariants of the particle motion is derived, and the condition for stochastic particle trajectories as well as the coefficients of particle diffusion in the three-dimensional particle invariant space are determined. Estimations of the diffusion coefficients are given in the two cases of waves interacting with passing and trapped ions by means of the cyclotron and bounce resonances, respectively. We also briefly discuss additional mechanisms which may destroy the regular particle motion along the bounce orbits in the presence of electromagnetic waves.

\section{Unperturbed particle motion}

We adopt the coordinate system $\left(x^{1}, x^{2}, x^{3}\right)$, where $x^{1}=$ const represents a magnetic surface, and $x^{2}=\theta$ and $x^{3}=\zeta$ are the poloidal and toroidal angular coordinates on that surface. We can then introduce an orthonormal basis

$$
\mathbf{e}_{a}=\nabla x^{1} /\left|\nabla x^{1}\right|, \quad \mathbf{e}_{b}=\mathbf{e}_{\|} \times \mathbf{e}_{a}, \quad \mathbf{e}_{\|}=\mathbf{B}_{0} / B_{0},
$$

with $\mathbf{B}_{0}$ the equilibrium magnetic field. Since all calculations in this work will be carried out for a large aspect ratio, axisymmetric tokamak with cirular cross section, the only nonzero metric components are given by

$$
g_{r r}=1, \quad g_{\theta \theta}=r^{2}, \quad g_{\zeta \zeta}=R^{2},
$$

with $R=R_{0}(1+\varepsilon \cos \theta)$ the major radius, $R_{0}$ the radial position of the magnetic axis, and $\varepsilon=r / R_{0} \ll 1$ the inverse aspect ratio. Then, the orthornormal basis vectors perpendicular to $\mathbf{B}_{0}$ can be approximated by

$$
\mathbf{e}_{a}=\mathbf{e}_{r}=\mathbf{e}^{r}, \quad \mathbf{e}_{b}=\mathbf{e}_{\|} \times \mathbf{e}_{a} \approx \frac{1}{r} \mathbf{e}_{\theta},
$$

and the background magnetic field can be written in the form $\mathbf{B}_{0}=B^{\theta} \mathbf{e}_{\theta}+B^{\zeta} \mathbf{e}_{\zeta}$, with $B^{\theta} \ll B^{\zeta}$. Hence,

$$
\mathbf{e}_{\|}=\frac{\mathbf{B}_{0}}{B_{0}} \approx \frac{1}{R}\left(\frac{1}{q} \mathbf{e}_{\theta}+\mathbf{e}_{\zeta}\right),
$$

where $q(r)$ is the safety factor, and by axisymmetry $B_{0}=$ $B_{\mathrm{s}}(1+\varepsilon \cos \theta)^{-1}$ with $B_{\mathrm{s}}$ the magnetic field strength at the magnetic axis.

In order to describe the particle trajectories in the absence of wave fields, we adopt the guiding center approximation, and write the particle position and velocity as $\mathbf{r}=\mathbf{r}_{\mathrm{gc}}+\mathbf{r}_{\mathrm{L}}$ and $\mathbf{v}=\mathbf{v}_{\mathrm{gc}}+\mathbf{v}_{\mathrm{L}}$. Here, $\mathbf{v}_{\mathrm{L}}=\mathrm{d} \mathbf{r}_{\mathrm{L}} / \mathrm{d} t$ is the Larmor rotation velocity and $\mathbf{v}_{\mathrm{gc}}=\mathrm{d} \mathbf{r}_{\mathrm{gc}} / \mathrm{d} t$ is the velocity of the guiding center, obtained by averaging over the Larmor motion. The Larmor gyration around the magnetic field lines is determined by the equation

$$
\frac{\mathrm{d} \mathbf{v}_{\mathrm{L}}}{\mathrm{d} t}=\frac{e}{m} \mathbf{v}_{\mathrm{L}} \times \mathbf{B}_{0}
$$

Transforming to curvilinear coordinates with basis vectors given by (3) and (4), we get

$$
v_{\mathrm{L}}^{a}=v_{\mathrm{L}} \cos \alpha, \quad v_{\mathrm{L}}^{b}=v_{\mathrm{L}} \sin \alpha,
$$

and it follows that

$$
\frac{\mathrm{d} \alpha}{\mathrm{d} t}=-\omega_{\mathrm{c}}
$$

where $\omega_{\mathrm{c}}$ is the cyclotron (Larmor) frequency. Hence,

$$
\alpha(t)=\alpha_{0}-\int_{0}^{t} \omega_{\mathrm{c}}\left(t^{\prime}\right) \mathrm{d} t^{\prime}
$$

and up to terms of $\mathcal{O}\left(r_{\mathrm{L}} / L\right)$, where $L$ is the length scale of the magnetic field inhomogeneity, we obtain

$$
\begin{aligned}
\mathbf{r}_{\mathrm{L}}(t)-\mathbf{r}_{\mathrm{L}}\left(t_{0}\right)= & \mathbf{e}_{a}\left[-\frac{v_{\mathrm{L}}}{\omega_{\mathrm{c}}(t)} \sin (\alpha(t))+\frac{v_{\mathrm{L}}}{\omega_{\mathrm{c}}\left(t_{0}\right)} \sin \left(\alpha\left(t_{0}\right)\right)\right] \\
& +\mathbf{e}_{b}\left[\frac{v_{\mathrm{L}}}{\omega_{\mathrm{c}}(t)} \cos (\alpha(t))-\frac{v_{\mathrm{L}}}{\omega_{\mathrm{c}}\left(t_{0}\right)} \cos \left(\alpha\left(t_{0}\right)\right)\right] .
\end{aligned}
$$

The guiding center velocity $\mathbf{v}_{\mathrm{gc}}$ is described by making the standard decomposition $\mathbf{v}_{\mathrm{gc}}=v_{\|} \mathbf{e}_{\|}+\mathbf{v}_{\mathrm{D}}$, where the drift velocity due to the magnetic field inhomogeneity and curvature is given by

$$
\mathbf{v}_{\mathrm{D}}=\frac{1}{2 \omega_{\mathrm{c}} B_{0}}\left(v_{\perp}^{2}+2 v_{\|}^{2}\right)\left(\mathbf{e}_{\|} \times \nabla B_{0}\right)=-v_{\|} \mathbf{e}_{\|} \times \nabla\left(\frac{v_{\|}}{\omega_{\mathrm{c}}}\right) .
$$

The covariant components of $\mathbf{v}_{\mathrm{gc}}$ then become

$$
\begin{gathered}
\frac{\mathrm{d} r}{\mathrm{~d} t}=\frac{v_{\|}}{r} \frac{\partial}{\partial \theta}\left(\frac{v_{\|}}{\omega_{\mathrm{c}}}\right), \\
\frac{\mathrm{d} \theta}{\mathrm{d} t}=\frac{v_{\|}}{q R}-\frac{v_{\|}}{r} \frac{\partial}{\partial r}\left(\frac{v_{\|}}{\omega_{\mathrm{c}}}\right), \\
\frac{\mathrm{d} \zeta}{\mathrm{d} t}=\frac{v_{\|}}{R} .
\end{gathered}
$$

The unperturbed particle motion in an axisymmetric magnetic field has two exact invariants: the particle kinetic energy

$$
W=\frac{m}{2}\left(v_{\|}^{2}+v_{\perp}^{2}\right),
$$

and the canonical angular momentum (see the appendix)

$$
P_{\zeta}=R\left(m v_{\zeta}+e A_{\zeta}\right)
$$

Moreover, the magnetic moment

$$
\mu=\frac{m v_{\perp}^{2}}{2 B_{0}}
$$

is an adiabatic invariant of particle motion. Using the expressions (12) and (14), the parallel particle velocity can be written as

$$
v_{\|}= \pm \sqrt{\frac{2}{m}\left(W-\mu B_{0}\right)}= \pm \frac{v_{\| 0}}{\kappa} \sqrt{\kappa^{2}-\sin ^{2} \frac{\theta}{2}},
$$

where

$$
v_{\| 0}=\left|v_{\|}(0)\right|=2 \kappa \sqrt{\frac{\mu B_{\mathrm{s}} \varepsilon}{m}} .
$$


Here, the trapping parameter $\kappa$ is defined by

$$
\kappa^{2}=\frac{W-\mu B_{\mathrm{s}}(1-\varepsilon)}{2 \mu B_{\mathrm{s}} \varepsilon},
$$

with $\kappa^{2}<1$ for trapped particles and $\kappa^{2}>1$ for passing particles. We now know $v_{\|}$as a function of $\theta$, and can then use equation $(11 b)$ to derive a relation between $t$ and $\theta$. Assuming that $q r_{\mathrm{L}} / r \ll \sqrt{\varepsilon}$, we get to lowest order

$$
t=\int_{0}^{t} \mathrm{~d} t^{\prime} \approx \frac{q R_{0} \kappa}{v_{\| 0}} \int_{\theta(0)}^{\theta(t)} \sqrt{\kappa^{2}-\sin ^{2} \frac{\theta}{2}} \mathrm{~d} \theta,
$$

where we have Taylor expanded around the average radial position $\bar{r} \equiv\langle r\rangle_{\mathrm{B}}$ of the periodic poloidal particle motion, and all quantities are understood to be calculated at $\bar{r}$. Here, the poloidal average, or simply bounce average, is defined as

$$
\langle\cdots\rangle_{\mathrm{B}} \equiv \frac{1}{\tau_{\mathrm{B}}} \int_{0}^{\tau_{\mathrm{B}}}(\cdots) \mathrm{d} t,
$$

where $\tau_{\mathrm{B}}$ is the period of the poloidal particle motion, the so-called bounce period, and $\omega_{\mathrm{B}}=2 \pi / \tau_{\mathrm{B}}$ is the bounce frequency.

The character of the integral (18) depends on whether $\kappa$ is larger or smaller than 1 . For passing particles $\left(\kappa^{2}>1\right)$, we use $\theta_{0}=-\pi$, and get

$$
t=\frac{2 q R_{0}}{v_{\| 0}}\left[K\left(\kappa^{-1}\right)+u\right],
$$

where

$$
u\left(\phi, \kappa^{-1}\right)=\int_{0}^{\phi} \frac{\mathrm{d} \alpha}{\sqrt{1-\kappa^{-2} \sin ^{2} \alpha}},
$$

$K\left(\kappa^{-1}\right)=u\left(\frac{\pi}{2}, \kappa^{-1}\right)$ is the complete elliptic integral of the first kind and $\theta=2 \phi=2 \operatorname{am}(u)$, with am the Jacobi amplitude. Thus, setting $t=\tau_{\mathrm{B}}$, we find the bounce period for passing particles to be

$$
\tau_{\mathrm{B}}=\frac{4 q R_{0} \kappa K\left(\kappa^{-1}\right)}{v_{\| 0}} .
$$

For trapped particles $\left(\kappa^{2}<1\right)$, we use $\theta_{0}=-\theta_{\mathrm{B}}$, with $\pm \theta_{\mathrm{B}}=$ $\pm 2 \arcsin \kappa$ the poloidal angles at which the trapped particles bounce. We then get

$$
t=\frac{2 q R_{0}}{v_{\| 0}}[K(\kappa)+u]
$$

where now

$$
u(\varphi, \kappa)=\int_{0}^{\varphi} \frac{\mathrm{d} \alpha}{\sqrt{1-\kappa^{2} \sin ^{2} \alpha}},
$$

and $\theta=2 \arcsin [\kappa \operatorname{sn}(u)]$, with $\operatorname{sn}(u)=\sin [\operatorname{am}(u)]$. The bounce period for the trapped particles is given by

$$
\tau_{\mathrm{B}}=\frac{8 q R_{0} \kappa K(\kappa)}{v_{\| 0}} .
$$

\section{Evolution equations for the invariants}

The present study is primarily concerned with the effect of a prescribed electromagnetic perturbation on the trajectory of a test particle. The electric and magnetic wave fields are represented as plane waves according to

$$
\left(\mathbf{E}_{1}, \mathbf{B}_{1}\right)=\left(\mathbf{E}_{k}, \mathbf{B}_{k}\right) \mathrm{e}^{-\mathrm{i} \omega t+\mathrm{ik} \cdot \mathbf{r}},
$$

where the amplitudes $\mathbf{E}_{k}$ and $\mathbf{B}_{k}$ are assumed to be small. Here, $\mathbf{k}$ is the wave vector and $\omega$ is the wave frequency. Since the poloidal and toroidal coordinates are both $2 \pi$-periodic, the $k_{2}$ and $k_{3}$ components of the wave vector must be integers $m$ and $n$, respectively. Using the orthonormal coordinate system (1), we can express the physical components of the wave field $\mathbf{E}_{1}$ and the wave vector $\mathbf{k}$ in the form

$$
\begin{gathered}
E_{a}=\mathbf{e}_{a} \cdot \mathbf{E}_{1}, \quad E_{b}=\mathbf{e}_{b} \cdot \mathbf{E}_{1}, \quad E_{\|}=\mathbf{e}_{\|} \cdot \mathbf{E}_{1}, \\
\mathrm{i} k_{a}=\mathbf{e}_{a} \cdot \nabla \ln E_{1}, \quad \mathrm{i} k_{b}=\mathbf{e}_{b} \cdot \nabla \ln E_{1}, \quad \mathrm{i} k_{\|}=\mathbf{e}_{\|} \cdot \nabla \ln E_{1} .
\end{gathered}
$$

Similar expressions can be written for the components of the vectors $\mathbf{v}$ and $\mathbf{B}_{1}$.

The appropriate equations describing the time evolution of the particle invariants $W, \mu$ and $P_{\zeta}$ due to the wave-particle interaction are derived in the appendix. The resulting expressions are given by

$$
\frac{\mathrm{d} W}{\mathrm{~d} t}=e \mathbf{v} \cdot \mathbf{E}_{k} \mathrm{e}^{-\mathrm{i} \omega t+\mathrm{ik} \cdot \mathbf{r}}
$$

$$
\frac{\mathrm{d}}{\mathrm{d} t}(\mu B)=e\left[\left(1-\frac{k_{\|} v_{\|}}{\omega}\right) \mathbf{v} \cdot \mathbf{E}_{k}-\left(1-\frac{\mathbf{k} \cdot \mathbf{v}}{\omega}\right) v_{\|} E_{\|}\right] \mathrm{e}^{-\mathrm{i} \omega t+\mathbf{i k} \cdot \mathbf{r}},
$$

$$
\frac{\mathrm{d} P_{\zeta}}{\mathrm{d} t}=e \frac{k_{\zeta} R}{\omega}\left[\mathbf{v} \cdot \mathbf{E}_{k}+(\omega-\mathbf{k} \cdot \mathbf{v}) \frac{\mathbf{k} \cdot \mathbf{E}_{k}}{k^{2}}\right] \mathrm{e}^{-\mathrm{i} \omega t+\mathrm{i} \mathbf{k} \cdot \mathbf{r}} .
$$

In order to determine the time evolution of the invariants we apply an approach based on the reduction of equations $(29 a)-(29 c)$ to a set of difference equations, which determine $\mathbf{I} \equiv\left(W, \mu, P_{\zeta}\right)$ for a particle in terms of its values one bounce period earlier. The procedure involves integrating equations (29a)-(29c) along the unperturbed particle trajectories derived in section 2 . We note that an alternative method for deriving the difference equations is based on the Hamilton formalism [1-6, 16, 17]. However, the number of phase space variables in the Hamiltonian approach is larger than that of the method presented here.

The time integration of $(29 a)-(29 c)$ is performed by expanding the invariants according to $\mathbf{I}=\mathbf{I}^{(0)}+\mathbf{I}^{(1)}+\ldots$ in the small wave amplitude $\mathbf{E}_{k}$. To lowest order, $\mathbf{I}$ is then a conserved quantity,

$$
\frac{\mathrm{d} \mathbf{I}^{(0)}}{\mathrm{d} t}=0,
$$

and to first order the non-adiabatic change in $\mathbf{I}$ is determined by equations (29a)-(29c), where it is now understood that $\mathbf{r}$ and $\mathbf{v}$ are the unperturbed (zeroth order) particle position and velocity, respectively. 
Introducing a constant angle $\chi$ such that $k_{a}=k_{\perp} \cos \chi$, $k_{b}=k_{\perp} \sin \chi$, and using the standard expansion

$$
\mathrm{e}^{-\mathrm{i} \xi \sin (\alpha-\chi)}=\sum_{l=-\infty}^{\infty} J_{l}(\xi) \mathrm{e}^{-\mathrm{i}(\alpha-\chi)},
$$

where $\xi=k_{\perp} v_{\mathrm{L}} / \omega_{\mathrm{c}}$, we obtain the first order non-adiabatic change in, for instance, $W$, during a bounce period $\tau_{\mathrm{B}}$ as

$$
\Delta W=e \sum_{l=-\infty}^{\infty} \mathrm{e}^{-\mathrm{i} \psi_{l 0}} \int_{0}^{\tau_{\mathrm{B}}} \mathbf{E}_{k} \cdot \mathbf{Q}_{l} \mathrm{e}^{-\mathrm{i} \psi_{l}(t)} \mathrm{d} t .
$$

Here, $\psi_{l 0}=l\left(\alpha_{0}-\chi\right)$, and the phase is given by

$$
\psi_{l}(t)=\int_{0}^{t} \mathrm{~d} t^{\prime}\left(\omega-l \omega_{\mathrm{c}}-\mathbf{k} \cdot \mathbf{v}_{\mathrm{gc}}\right) .
$$

Furthermore,

$$
\mathbf{Q}_{l}=\left(\left(\frac{l}{\xi} v_{\mathrm{L}}+v_{\mathrm{D} 1}\right) J_{l}, i v_{\mathrm{L}} J_{l}^{\prime}+v_{\mathrm{D} 2} J_{l}, v_{\|} J_{l}\right),
$$

where the primes denote derivatives with respect to $\xi$, and we have transformed to a system where coordinates are labeled $1,2,3$, and vector components are given by

$$
A_{1}=\frac{\mathbf{k}_{\perp}}{k_{\perp}} \cdot \mathbf{A}, \quad A_{2}=\left[\frac{\mathbf{k}_{\perp}}{k_{\perp}} \times \mathbf{A}\right]_{\|}, \quad A_{3}=A_{\|} .
$$

The physical meaning of the representation (32) is that in cylindrical geometry in velocity space the plane wave consists of a sum of cyclotron harmonics, and the particle interacts with each of them. Besides, by separating the fast and slow variations according to (31), it will be possible to apply the stationary phase method to evaluate time integrals of the type (32), with the stationary points determined by the condition

$$
\frac{\mathrm{d} \psi_{l}}{\mathrm{~d} t}=\omega-l \omega_{\mathrm{c}}-k_{\|} v_{\|}-\mathbf{k}_{\perp} \cdot \mathbf{v}_{\mathrm{D}}=0 .
$$

However, the calculations have to be done for passing and trapped particles separately. For simplicity, we assume in the rest of the paper that $k_{r} v_{\mathrm{D}} \ll \omega$.

\subsection{Passing particles $\kappa^{2}>1$}

Using the relations (20)-(22), we can rewrite $\Delta W$ as

$$
\Delta W=\sum_{l=-\infty}^{\infty} \Delta W_{l},
$$

where each harmonic contribution is given by

$$
\Delta W_{l}=\frac{e}{\omega_{\mathrm{B}}} \mathrm{e}^{-\mathrm{i}\left[\psi_{l}(\pi)+\psi_{l 0}\right]} \int_{-\pi}^{\pi} \mathbf{E}_{k} \cdot \mathbf{Q}_{l} \mathrm{e}^{-\mathrm{i} \psi_{l}} \mathrm{~d} \tau .
$$

Here, the integration variable is $\tau=\frac{\pi}{K\left(\kappa^{-1}\right)} u$, and we have

$$
\begin{aligned}
\psi_{l}= & \frac{2 q R_{0}}{v_{\| 0}} \Delta \omega u-2 \lambda \operatorname{am}(u)-2 n q \varepsilon \operatorname{cn}(u) \operatorname{sn}(u)+\frac{\kappa^{2} \pi}{\omega_{\mathrm{B}} K\left(\kappa^{-1}\right)} \\
& \times\left[G_{\mathrm{p}} Z(u)-n \omega_{\mathrm{D}} \frac{2}{3} \frac{\varepsilon}{1+2 \varepsilon \kappa^{2}} \operatorname{cn}(u) \operatorname{dn}(u) \operatorname{sn}(u)\right]
\end{aligned}
$$

with

$$
\begin{gathered}
\psi_{l}(\pi)=\left(\frac{\Delta \omega}{\omega_{\mathrm{B}}}-\lambda\right) \pi \\
\Delta \omega=\omega-l\left\langle\omega_{\mathrm{c}}\right\rangle_{\mathrm{B}}-n\left\langle\omega_{\zeta}\right\rangle_{\mathrm{B}} \\
Z(u)=\int_{0}^{u} \mathrm{dn}^{2}\left(u^{\prime}\right) \mathrm{d} u^{\prime}-\frac{E\left(\kappa^{-1}\right)}{K\left(\kappa^{-1}\right)} u \\
G_{\mathrm{p}}=2 l \varepsilon \omega_{\mathrm{cs}}-n \omega_{\mathrm{D}}\left[1-\frac{2}{3} \frac{\varepsilon}{1+2 \varepsilon \kappa^{2}}\left(\frac{1}{2}-\kappa^{2}\right)+\frac{2 s}{1+2 \varepsilon \kappa^{2}}\right],
\end{gathered}
$$

and

$\lambda=q R_{0} k_{\|}, \quad \omega_{\mathrm{cs}}=\frac{e B_{\mathrm{s}}}{m}, \quad \omega_{\mathrm{D}}=-\frac{2 q W}{r R_{0} m \omega_{\mathrm{cs}}}, \quad s=\frac{r}{q} \frac{\mathrm{d} q}{\mathrm{~d} r}$.

In these expressions, am and $\mathrm{dn}$ are Jacobian elliptic functions (more precisely the Jacobian amplitude and the delta amplitude) and $K$ and $E$ are the complete elliptical integrals of the first and second kind, respectively. Applying the definition (19), the bounce averages of $\omega_{\mathrm{c}}$ and $\omega_{\zeta}$ are given by

$$
\begin{gathered}
\left\langle\omega_{\mathrm{c}}\right\rangle_{\mathrm{B}}=\omega_{\mathrm{cs}}\left\{1-\varepsilon\left[1-2 \kappa^{2}\left(1-\frac{E\left(\kappa^{-1}\right)}{K\left(\kappa^{-1}\right)}\right)\right]\right\}, \\
\left\langle\omega_{\zeta}\right\rangle_{\mathrm{B}}=\omega_{\mathrm{D}}\left[G_{1}+2 s G_{2}\right],
\end{gathered}
$$

where

$$
\begin{aligned}
& G_{1}= \frac{1}{2}-\kappa^{2}\left[1-\frac{E\left(\kappa^{-1}\right)}{K\left(\kappa^{-1}\right)}\right] \\
&+\frac{2}{3} \frac{\varepsilon \kappa^{2}}{1+2 \varepsilon \kappa^{2}}\left[1-\kappa^{2}-\left(\frac{1}{2}-\kappa^{2}\right) \frac{E\left(\kappa^{-1}\right)}{K\left(\kappa^{-1}\right)}\right], \\
& G_{2}=\frac{\kappa^{2}}{1+2 \varepsilon \kappa^{2}} \frac{E\left(\kappa^{-1}\right)}{K\left(\kappa^{-1}\right)}\left[1-\frac{\pi^{2}}{4 K\left(\kappa^{-1}\right) E\left(\kappa^{-1}\right)}\right] .
\end{aligned}
$$

Note that all quantities in the above expressions are calculated at the average radial particle position $\bar{r}$, and that we have neglected terms of $\mathcal{O}\left(\varepsilon^{2}\right)$.

We now apply the stationary phase method on all the harmonic contributions $\Delta W_{l}$ by expanding the phase $\psi_{l}$ around each of its stationary points according to

$$
\psi_{l}=\psi_{l r}+\frac{\sigma}{2}\left|\ddot{\psi}_{l r}\right|\left(\tau-\tau_{r}\right)^{2} .
$$

The subscript $r$ denotes quantities evaluated at the stationary (resonant) points, and we have $\psi_{l r}=\psi\left(\tau_{r}\right), \sigma=\operatorname{sign}\left(\ddot{\psi}_{l r}\right)$, and

$$
\left.\ddot{\psi}_{l r} \equiv \frac{\mathrm{d}^{2} \psi_{l}}{\mathrm{~d} \tau^{2}}\right|_{\tau=\tau_{r}}=\left.\left(\frac{K\left(\kappa^{-1}\right)}{\pi}\right)^{2} \frac{\mathrm{d}^{2} \psi_{l}}{\mathrm{~d} u^{2}}\right|_{u=u_{r}} .
$$

The expression $\dot{\psi}_{l}=0$ determining the stationary points is a fourth order algebraic equation for the even function $\operatorname{dn}(u)$. In general, there are then eight stationary points of the passing particle motion. The analysis can, however, be made more explicit by neglecting the effect of the particle precessional 


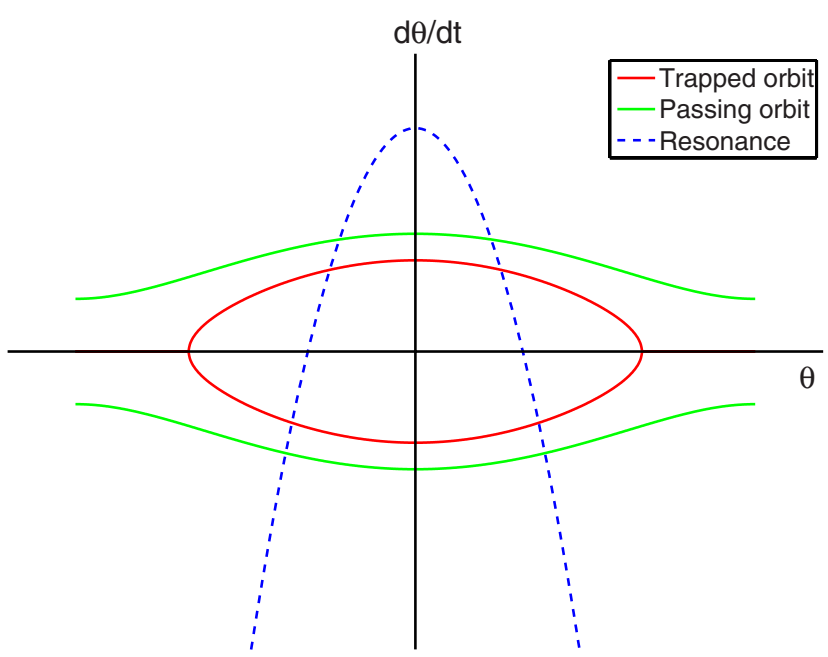

Figure 1. Case (a): qualitative illustration of the resonance conditions for passing and trapped particles when $\omega \gg\left|\mathbf{k} \cdot \mathbf{v}_{\mathrm{D}}\right|$ and $l \neq 0, k_{\|} \neq 0$.

motion, i.e. in the limit $\omega \gg\left|\mathbf{k} \cdot \mathbf{v}_{\mathrm{D}}\right|$. Then, the resonant points are given by

$$
\Delta \omega-v_{\| 0}\left[k_{\|}+\frac{n \varepsilon}{R_{0}}\right] \operatorname{dn}(u)+\kappa^{2} G_{\mathrm{p}}\left[\operatorname{dn}^{2}(u)-\frac{E\left(\kappa^{-1}\right)}{K\left(\kappa^{-1}\right)}\right]=0,
$$

where we have neglected small terms of $\mathcal{O}\left(\varepsilon / \kappa^{2}\right)$. The solution to (51) is

$$
\operatorname{dn}\left( \pm u_{r}\right)=d_{\mathrm{p}}+\left[d_{\mathrm{p}}^{2}+\frac{E\left(\kappa^{-1}\right)}{K\left(\kappa^{-1}\right)}-\frac{\Delta \omega}{G_{\mathrm{p}}}\right]^{1 / 2},
$$

where $d_{\mathrm{p}}=\frac{v_{\| 0}}{2 \kappa^{2} G_{\mathrm{p}}}\left(k_{\|}+n \varepsilon / R_{0}\right)$. In the particular case when $l=0$, we get

$$
\operatorname{dn}\left( \pm u_{r}\right)=\frac{\omega}{v_{\| 0}\left(k_{\|}+n \varepsilon / R_{0}\right)}
$$

Figures 1-3 illustrate qualitatively the resonance conditions (for both passing and trapped particles) under the assumption $\omega \gg\left|\mathbf{k} \cdot \mathbf{v}_{\mathrm{D}}\right|$ and in situations when: (a) $l \neq 0, k_{\|} \neq 0$; (b) $l \neq 0$, $k_{\|}=0$; (c) $l=0, k_{\|} \neq 0$. As can be seen, passing particles always interact with the wave and there exist two resonant points.

Finally, adding the contributions to $\Delta W_{l}$ from the two stationary points obtained in the limit $\omega \gg\left|\mathbf{k} \cdot \mathbf{v}_{\mathrm{D}}\right|$ yields

$$
\Delta W_{l}=\mathrm{e}^{-\mathrm{i}\left[\psi_{l 0}+\psi_{l}(\pi)\right]} \alpha_{W} \cos \left(\psi_{l r}+\sigma \frac{\pi}{4}\right),
$$

where

$$
\begin{gathered}
\alpha_{W}=\frac{2 e}{\omega_{\mathrm{B}}} \sqrt{\frac{2 \pi}{\left|\ddot{\psi}_{l r}\right|}} \mathbf{Q}_{l} \cdot \mathbf{E}_{k}, \\
\mathbf{Q}_{l}=\left(\frac{l}{\xi} v_{\mathrm{L}} J_{l}, \mathrm{i} v_{\mathrm{L}} J_{l}^{\prime}, v_{\| 0} \operatorname{dn}\left(u_{r}\right) J_{l}\right),
\end{gathered}
$$

and we have used that $\psi_{l}(\tau)=-\psi_{l}(-\tau)$.

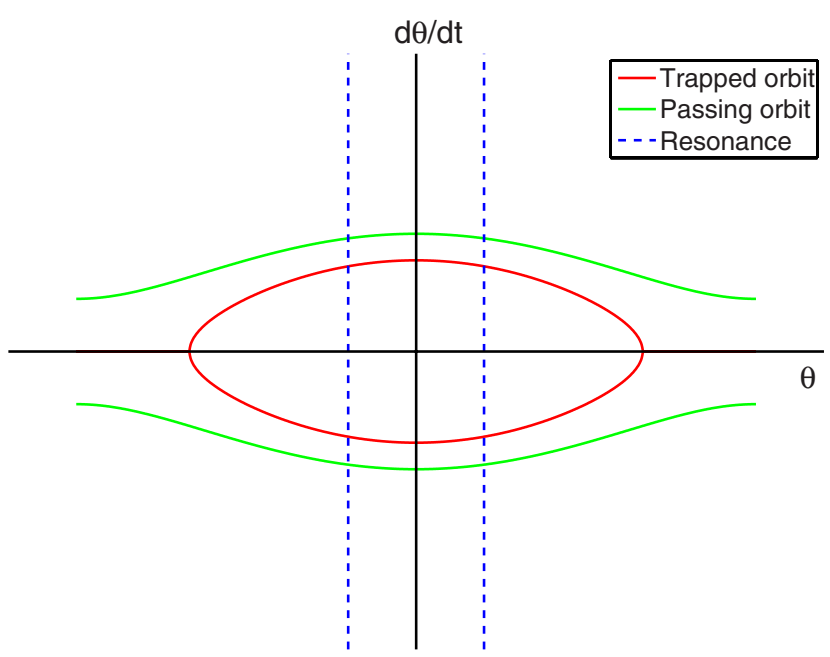

Figure 2. Case (b): qualitative illustration of the resonance conditions for passing and trapped particles when $\omega \gg\left|\mathbf{k} \cdot \mathbf{v}_{\mathrm{D}}\right|$ and $l \neq 0, k_{\|}=0$.

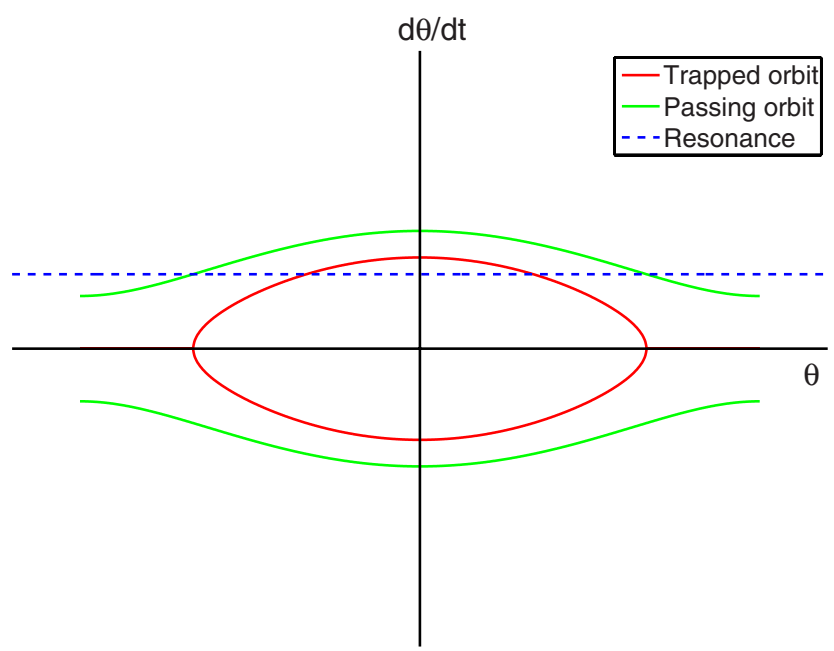

Figure 3. Case (c): qualitative illustration of the resonance conditions for passing and trapped particles when $\omega \gg\left|\mathbf{k} \cdot \mathbf{v}_{\mathrm{D}}\right|$ and $l=0, k_{\|} \neq 0$.

\subsection{Trapped particles $\kappa^{2}<1$}

For trapped particles, we use the relations in equations (23)-(25), and write $\Delta W$ once more in the forms (37) and (38), where now the integration variable is $\tau=\frac{\pi}{K(\kappa)} u$. We then have

$$
\begin{aligned}
\psi_{l}= & \frac{2 q R_{0} \kappa}{v_{\| 0}} \Delta \omega u-2 \lambda \arcsin [\kappa \operatorname{sn}(u)] \\
& +\frac{\pi G_{\mathrm{t}}}{2 \omega_{\mathrm{B}} K(\kappa)} Z(u)-2 n \varepsilon q \kappa \operatorname{dn}(u) \operatorname{sn}(u),
\end{aligned}
$$

with

$$
\psi_{l}(\pi)=\frac{\Delta \omega}{\omega_{\mathrm{B}}} \frac{\pi}{2}-2 \lambda \arcsin \kappa-2 n \varepsilon q \kappa \sqrt{1-\kappa^{2}},
$$

and $\Delta \omega=\omega-l\left\langle\omega_{\mathrm{c}}\right\rangle_{\mathrm{B}}-n\left\langle\omega_{\zeta}\right\rangle_{\mathrm{B}}$ as for passing particles. In these expressions,

$$
Z(u)=\int_{0}^{u} \operatorname{dn}^{2}\left(u^{\prime}\right) \mathrm{d} u^{\prime}-\frac{E(\kappa)}{K(\kappa)} u
$$


and

$$
G_{\mathrm{t}}=2 l \varepsilon \omega_{\mathrm{cs}}-n \omega_{\mathrm{D}}[1+2 s],
$$

and the bounce averaged frequencies are given by

$$
\begin{gathered}
\left\langle\omega_{\mathrm{c}}\right\rangle_{\mathrm{B}}=\omega_{\mathrm{cs}}\left\{1+\varepsilon\left[1-2 \frac{E(\kappa)}{K(\kappa)}\right]\right\}, \\
\left\langle\omega_{\zeta}\right\rangle_{\mathrm{B}}=n \omega_{\mathrm{D}}\left\{\frac{E(\kappa)}{K(\kappa)}-\frac{1}{2}+2 s\left[\frac{E(\kappa)}{K(\kappa)}+\kappa^{2}-1\right]\right\} .
\end{gathered}
$$

As usual, all quantities have been calculated at the average radial position $\bar{r}$ during the poloidal bounce motion, and we have neglected small terms of $\mathcal{O}\left(\varepsilon \kappa^{2}\right)$.

Applying once more the stationary phase method, we approximate $\psi_{l}$ around its minima by (49). The resonant points are then determined by

$$
\begin{aligned}
& \Delta \omega-v_{\| 0}\left[k_{\|}+\frac{n \varepsilon}{R_{0}}\right] \operatorname{cn}(u) \\
& +G_{\mathrm{t}}\left[1-\kappa^{2}-\frac{E(\kappa)}{K(\kappa)}+\kappa^{2} \mathrm{cn}^{2}(u)\right]=0,
\end{aligned}
$$

where we have neglected terms of $\mathcal{O}\left(\varepsilon \kappa^{2}\right)$. Since $\operatorname{cn}(u)$ is even, there are in general four solutions to (63), given by

$$
\operatorname{cn}\left( \pm u_{r}\right)=d_{t} \pm\left[d_{t}^{2}-\frac{1}{\kappa^{2}}\left(1-\kappa^{2}-\frac{E(\kappa)}{K(\kappa)}\right)-\frac{\Delta \omega}{\kappa^{2} G_{\mathrm{t}}}\right]^{1 / 2},
$$

with $d_{t}=\frac{v_{\| 0}}{2 \kappa^{2} G_{\mathrm{t}}}\left(k_{\|}+n \varepsilon / R_{0}\right)$. Figures 1-3 illustrate that for trapped particles, the interaction is indeed characterized by four resonant points. Adding the contributions from each of these points and invoking the limit $\omega \gg\left|\mathbf{k} \cdot \mathbf{v}_{\mathrm{D}}\right|$ yields

$$
\Delta W_{l}=\mathrm{e}^{-\mathrm{i}\left[\psi_{l 0}+\psi_{l}(\pi)\right]} \sum_{ \pm} \alpha_{W}^{ \pm} \cos \left(\psi_{l r}^{ \pm}+\sigma_{ \pm} \frac{\pi}{4}\right)
$$

where

$$
\begin{gathered}
\alpha_{W}^{ \pm}=\frac{2 e}{\omega_{\mathrm{B}}} \sqrt{\frac{2 \pi}{\left|\ddot{\psi}_{l r}^{ \pm}\right|}} \mathbf{Q}_{l}^{ \pm} \cdot \mathbf{E}_{k}, \\
\mathbf{Q}_{l}^{ \pm}=\left(\frac{l}{\xi} v_{\mathrm{L}} J_{l}, \mathrm{i} v_{\mathrm{L}} J_{l}^{\prime}, v_{\| 0} \operatorname{cn}\left(u_{r}^{ \pm}\right) J_{l}\right) .
\end{gathered}
$$

Here, \pm labels the two pairs of stationary points corresponding to the \pm in the right hand side of (64). Note that in the special case when $l=0$, there are only two resonant points (see figure 3 ), determined by

$$
\operatorname{cn}\left( \pm u_{r}\right)=\frac{\omega}{v_{\| 0}\left(k_{\|}+n \varepsilon / R_{0}\right)} \text {. }
$$

The obtained expressions for $\Delta W$ determine the non-adiabatic change in the particle kinetic energy for passing and trapped particles. Non-adiabatic changes in the magnetic moment $\mu$ and the canonical angular momentum $P_{\zeta}$ follow from equations (29b) and (29c), respectively. Since the term containing $\omega-\mathbf{k} \cdot \mathbf{v}$ in $(29 b)$ is of the order $\left|\ddot{\psi}_{l r}\right|^{-3 / 2}$ at the resonance it can be neglected, and the similar term in (29c) vanishes identically due to the time integration. The calculations of the first order changes $\Delta \mu$ and $\Delta P_{\zeta}$ are then very similar to those leading to $\Delta W$, with the only effect being that the coefficients $\alpha_{W}$ and $\alpha_{W}^{ \pm}$are altered. In the limit $\omega \gg\left|\mathbf{k} \cdot \mathbf{v}_{\mathrm{D}}\right|$, we have

$$
\alpha_{\mu}^{ \pm}=\frac{l \omega_{\mathrm{cs}}}{\omega B_{\mathrm{s}}} \alpha_{W}^{ \pm}, \quad \alpha_{P_{\zeta}}^{ \pm}=\frac{k_{\zeta} R_{0}}{\omega} \alpha_{W}^{ \pm},
$$

where the notation \pm is to be ignored for passing particles. Note that in general, $\alpha_{\mu}^{ \pm}$and $\alpha_{P_{\zeta}}^{ \pm}$also contains contributions due to the particle drifts, and that these are omitted here by invoking the limit $\omega \gg\left|\mathbf{k} \cdot \mathbf{v}_{\mathrm{D}}\right|$. We finally note that the stationary phase approximation is valid if the time of resonant interaction is much less than the period of the poloidal particle motion. This is satisfied under the condition $\left|\ddot{\psi}_{l}\right| \gg 1$.

\section{Stochastic nature of the wave-particle interaction}

Using the expressions for $\Delta W, \Delta \mu$ and $\Delta P_{\zeta}$, we can trace the motion of a particle on the phase space with coordinates $W, \mu$ and $P_{\zeta}$ by examining the value of $\mathbf{I} \equiv\left(W, \mu, P_{\zeta}\right)$ at discrete times, separated by intervals of the bounce period $\tau_{\mathrm{B}}$. A set of finite difference mapping equations, connecting $\mathbf{I}$ and $\widetilde{\psi}_{l} \equiv \psi_{l 0}+\psi_{l}(\pi)$ for each harmonic from one bounce point to the next, can be written as

$$
\begin{gathered}
\mathbf{I}_{j+1}=\mathbf{I}_{j}+\mathrm{e}^{\mathrm{i} \widetilde{\psi}_{j}} \vec{\alpha}_{l} \cos \left(\psi_{l r}+\frac{\pi}{4}\right), \\
\widetilde{\psi}_{j+1}=\widetilde{\psi}_{j}+2 \psi_{l}(\pi),
\end{gathered}
$$

for a passing orbit, and as

$$
\begin{gathered}
\mathbf{I}_{j+1}=\mathbf{I}_{j}+\mathrm{e}^{\mathrm{i} \widetilde{\psi}_{j}}\left[\vec{\alpha}_{l}^{+} \cos \left(\psi_{l r}^{+}+\frac{\pi}{4}\right)+\vec{\alpha}_{l}^{-} \cos \left(\psi_{l r}^{-}+\frac{\pi}{4}\right)\right], \\
\widetilde{\psi}_{j+1}=\widetilde{\psi}_{j}+2 \psi_{l}(\pi),
\end{gathered}
$$

for a trapped orbit. Here,

$$
\vec{\alpha}_{l}^{ \pm}=\left(\alpha_{W}^{ \pm}, \alpha_{\mu}^{ \pm}, \alpha_{P_{\zeta}}^{ \pm}\right)
$$

and $\psi_{l}(\pi)$ is given by (40) for passing particles and (58) for trapped particles. The mapping equations (70a) and (70b) and $(71 a)$ and $(71 b)$ are written for the resonant points characterized by $\ddot{\psi}_{l r}>0$, and we have suppressed the index $l$ on $\widetilde{\psi}_{l, j}$ in order to keep the notation clear.

Since the functions $\psi_{l}(\pi)$ and $\psi_{l r}$ depend on $\mathbf{I}$, the mapping equations represent a coupled system of nonlinear equations. The strongest interaction takes place if $\psi_{l}(\pi)=$ $s \pi$, with $s=0, \pm 1, \pm 2, \ldots$, which can be written as

$$
\Delta \omega=(s+\lambda) \omega_{\mathrm{B}} \approx\left(k_{\|}+\frac{s}{q R_{0}}\right) v_{\| 0}
$$

for passing particles and

$$
\Delta \omega=\left(2 s+\frac{4}{\pi} \lambda \arcsin \kappa+\frac{4}{\pi} n \varepsilon q \kappa \sqrt{1-\kappa^{2}}\right) \omega_{\mathrm{B}}
$$

for trapped particles. Equations (73) and (74) define a set of resonant surfaces in I-space. Since the nonlinear terms in equations (70a) and (70b) and (71a) and (71b) are harmonic functions, their solutions should oscillate around the resonant surfaces, and the period and amplitude of these oscillations will depend on the value of the coupling coefficients $\vec{\alpha}$. Thus, although non-adiabatic interaction between the wave 
and particles occurs, equations (70a) and (70b) and (71a) and (71b) describe a regular time evolution of I (superadiabaticity) if the wave amplitude is below a certain threshold. The transition from superadiabatic to stochastic behaviour occurs for sufficiently large values of $\vec{\alpha}$, when the resonances overlap in phase space.

In order to obtain an approximate criterion for the onset of stochasticity, we consider a point $\mathbf{I}_{\mathrm{s}}$ on the resonant surface and expand $\psi_{l}(\pi)$ in the vicinity of this point:

$$
\psi_{l}(\pi)=s \pi+\left[\mathbf{I}_{j+1}-\mathbf{I}_{\mathrm{s}}\right] \cdot\left[\frac{\partial \psi_{l}(\pi)}{\partial \mathbf{I}_{j+1}}\right]_{\mathbf{I}_{j+1}=\mathbf{I}_{\mathrm{s}}} .
$$

Introducing the new variables $\tilde{I}_{j, n}=2 \frac{\partial \psi_{l}(\pi)}{\partial I_{\mathrm{s}, n}}\left(I_{j, n}-I_{\mathrm{s}, n}\right)$, where the index $n$ runs over the coordinates $\mathbf{I}$, the system of mapping equations may be rewritten as

$$
\begin{gathered}
\tilde{\mathbf{I}}_{j+1}=\tilde{\mathbf{I}}_{j}+\mathbf{K} \mathrm{e}^{\mathrm{i} \widetilde{\psi}_{j}}, \\
\tilde{\psi}_{j+1}=\tilde{\psi}_{j}+\sum_{n=1}^{3} \tilde{I}_{j+1, n},
\end{gathered}
$$

where

$$
K_{n}=2 \frac{\partial \psi_{l}(\pi)}{\partial I_{\mathrm{s}, n}} \alpha_{n} \cos \left(\psi_{l r}\left(\mathbf{I}_{\mathrm{s}}\right)+\frac{\pi}{4}\right)
$$

for passing particles, and

$$
K_{n}=2 \frac{\partial \psi_{l}(\pi)}{\partial I_{\mathrm{s}, n}}\left[\alpha_{n}^{+} \cos \left(\psi_{l r}^{+}\left(\mathbf{I}_{\mathrm{s}}\right)+\frac{\pi}{4}\right)+\alpha_{n}^{-} \cos \left(\psi_{l r}^{-}\left(\mathbf{I}_{\mathrm{s}}\right)+\frac{\pi}{4}\right)\right]
$$

for trapped particles. In order to investigate the system (76a) and $(76 b)$ more closely, we add the three equations $(76 a)$ and obtain

$$
\begin{gathered}
\bar{I}_{j+1}=\bar{I}_{j}+K_{e} \mathrm{e}^{\mathrm{i} \widetilde{\psi}_{j}}, \\
\widetilde{\psi}_{j+1}=\widetilde{\psi}_{j}+\bar{I}_{j+1},
\end{gathered}
$$

where $\bar{I}_{j}=\tilde{I}_{j, 1}+\tilde{I}_{j, 2}+\tilde{I}_{j, 3}$, and $K_{e}=K_{1}+K_{2}+K_{3}$. The system of equations (79a) and (79b) has the same structure as the 1D standard mapping [5]. Therefore, we can by analogy conclude that the particle motion is stochastic for $\left|K_{e}\right|>1$, and superadiabatic for $K_{e} \simeq 1$. Since $K_{e}$ is a function of $\mathbf{I}$, the condition $K_{e} \simeq 1$ determines a phase space stochasticity boundary. In the stochastic regime, i.e. when the wave amplitude is above the threshold value for stochastic motion, the process is Markovian, and the diffusion coefficients in the appropriate Fokker-Planck equation can be calculated as

$$
D\{\Delta \mathbf{I}, \Delta \mathbf{I}\}=\langle\Delta \mathbf{I} \cdot \Delta \mathbf{I}\rangle \sim \frac{1}{2} \vec{\alpha} \cdot \vec{\alpha}^{*},
$$

where the bra-ket notation denotes an average over the wave phase at the resonant point. In (80), it is necessary to specify the wave characteristics, i.e. the polarization, frequency and wave vector.

Let us estimate the particle energy diffusion coefficients in the two cases of waves interacting with passing and trapped ions by means of the cyclotron and bounce resonances, respectively. In the first case, considering quasi-linear diffusion without bounce resonances and assuming well passing particles, we obtain

$$
D=\frac{4 \pi e^{2} \mu B_{\mathrm{s}}}{m^{2} \tau_{\mathrm{B}}} \sum_{l}\left|\ddot{\psi}_{l r}\right|^{-1}\left[J_{l-1}^{2}\left|E_{+}\right|^{2}+J_{l+1}^{2}\left|E_{-}\right|^{2}\right],
$$

where $E_{ \pm}=\frac{1}{2}\left(E_{1} \pm E_{2}\right)$. This expression is in agreement with the result obtained in [11]. In the second case we consider the interaction between well trapped particles and a wave having frequency much lower than the cyclotron frequency but much larger than the particle precession frequency, and with the electric field and wave vector pointing along the magnetic field. Here, the velocity diffusion coefficient can be estimated as

$$
D=\frac{2 \pi e^{2}\left|E_{\|}\right|^{2} v_{\| 0} s^{2}}{m^{2} k_{\|} \lambda^{2} \kappa^{2}}
$$

which coincides with the result of [13].

Finally, we briefly discuss additional mechanisms which may destroy the regular particle motion along the bounce orbits in the presence of wave fields. The first mechanism is associated with Coulomb collisions of particles. The effect of collisions may be taken into account in the difference equations (70a) and (70b) and (71a) and (71b) by adding an appropriate term in the phase equations [12]. Assuming that the trapping parameter can be written as $\kappa^{2}=\bar{\kappa}^{2}+\widetilde{\kappa}^{2}$, where $\bar{\kappa}$ and $\widetilde{\kappa}$ are the regular and non-regular (connected with collisions) parts of $\kappa$, respectively, the new term appearing in the phase equations is $\left[\mathrm{d} \psi_{l}(\pi) / \mathrm{d} \kappa^{2}\right] \widetilde{\kappa}^{2}$. Collisions will sufficiently change the phase if

$$
\left[\frac{\mathrm{d} \psi_{l}(\pi)}{\mathrm{d} \kappa^{2}}\right]\left\langle\widetilde{\kappa}^{2}\right\rangle \simeq \frac{\pi}{2}
$$

Here, $\left\langle\widetilde{\kappa}^{2}\right\rangle$ is the dispersion of $\widetilde{\kappa}^{2}$ during the bounce period $\tau_{\mathrm{B}}$, i.e.

$$
\left\langle\left(\kappa^{2}\right)^{2}\right\rangle \simeq v_{*} \tau_{b}
$$

where $\nu_{*}$ is the effective collision frequency. For trapped particles, $v_{*} \simeq v / \sqrt{\varepsilon}$, and for passing particles, $v_{*} \simeq v$. Substituting (84) into (83) and estimating $\mathrm{d} \psi_{l}(\pi) / \mathrm{d} \kappa^{2}$, we find that in the case when $\omega \gg\left|\mathbf{k} \cdot \mathbf{v}_{\mathrm{D}}\right|$, the effect of collisions is small if

$$
\begin{gathered}
\frac{l \varepsilon \omega_{\mathrm{cs}}}{\omega_{\mathrm{B}}}\left(v_{*} \tau_{\mathrm{B}}\right)^{1 / 2} \ll 1, \quad l \neq 0, \\
\frac{\omega}{\omega_{\mathrm{B}}}\left(v_{*} \tau_{\mathrm{B}}\right)^{1 / 2} \ll 1, \quad l=0 .
\end{gathered}
$$

Since $\omega_{\mathrm{B}} \ll \omega_{\mathrm{cs}}$, so that the particles complete a large number of Larmor orbits between the resonant points, the conditions (85a) and (85b) are satisfied for sufficiently small values of $v_{*}$. However, a relatively small distortion of the toroidal symmetry of the equilibrium magnetic field can lead to a large effective collision frequency and may result in collisional particle diffusion. In tokamaks, this situation arises in the presence of a toroidal magnetic field ripple $\delta \omega_{\mathrm{cs}} \cos (N \zeta)$, where $\delta$ is the relative amplitude of the field 
ripple and $N$ is the number of toroidal field coils. Actually, the effective collision frequency is large, $v_{*}=\frac{N^{2} q^{2}}{2 \varepsilon} \nu \gg v$, and the diffusion of banana trapped particles can take place even in the case of very small pitch-angle scattering. According to $(85 a)$ and $(85 b)$, the ripple effects may be neglected if

$$
\delta \ll \sqrt{\frac{\varepsilon \omega_{\mathrm{cs}}}{v N^{2} q^{2}}}\left(\frac{\rho}{q R_{0}}\right)^{3 / 2}
$$

where

$$
\rho=\frac{\sqrt{\mu B_{\mathrm{s}} \varepsilon / m}}{\omega_{\mathrm{cs}}}
$$

\section{Appendix. Derivation of the evolution equations}

To derive equations describing the evolution of $W$ and $\mu$ in the presence of an electromagnetic wave, we start from the equation of motion for a charged particle, i.e.

$$
m \frac{\mathrm{d} \mathbf{v}}{\mathrm{d} t}=q\left[\mathbf{E}_{1}+\mathbf{v} \times\left(\mathbf{B}_{0}+\mathbf{B}_{1}\right)\right],
$$

where $\mathbf{B}_{0}$ is the equilibrium magnetic field and $\mathbf{E}_{1}$ and $\mathbf{B}_{1}$ are the wave fields given by equation (26). By means of Faraday's law

$$
\nabla \times \mathbf{E}_{1}=-\frac{\partial \mathbf{B}_{1}}{\partial t},
$$

the equation of motion (A.1) can be rewritten as

$$
m \frac{\mathrm{d} \mathbf{v}}{\mathrm{d} t}=\frac{q}{c} \mathbf{v} \times \mathbf{B}_{0}+q\left[\left(1-\frac{\mathbf{k} \cdot \mathbf{v}}{\omega}\right) \mathbf{E}_{k}+\frac{\mathbf{k}\left(\mathbf{v} \cdot \mathbf{E}_{k}\right)}{\omega}\right] \mathrm{e}^{-\mathrm{i} \omega t+\mathbf{i k} \cdot \mathbf{r}} .
$$

The equations describing the evolution of $W$ and $\mu$ due to the wave-particle interaction are now obtained by taking the scalar product of equation (A.3) with the vectors $\mathbf{v}$ and $\mathbf{v}_{\perp}$, respectively. The results are

$$
\frac{\mathrm{d} W}{\mathrm{~d} t}=e \mathbf{v} \cdot \mathbf{E}_{k} \mathrm{e}^{-\mathrm{i} \omega t+\mathrm{i} \mathbf{k} \cdot \mathbf{r}}
$$

and

$$
\frac{\mathrm{d}}{\mathrm{d} t}\left(\mu B_{0}\right)=e\left[\left(1-\frac{k_{\|} v_{\|}}{\omega}\right) \mathbf{v} \cdot \mathbf{E}_{k}-\left(1-\frac{\mathbf{k} \cdot \mathbf{v}}{\omega}\right) v_{\|} E_{\|}\right] \mathrm{e}^{-\mathrm{i} \omega t+\mathbf{i k} \cdot \mathbf{r}}
$$

In order to derive an equation describing the time evolution of $P_{\zeta}$, one has to resort to Lagrangian mechanics. The charged particle Lagrangian is given by

$$
\mathcal{L}=\frac{m}{2} \dot{\mathbf{r}} \cdot \dot{\mathbf{r}}+e \dot{\mathbf{r}} \cdot \mathbf{A}-e \phi
$$

where the scalar and vector potentials $\phi$ and $\mathbf{A}$ are functions of $\mathbf{r}$ and $t$ only, satisfying

$$
\mathbf{E}=-\nabla \phi-\frac{\partial \mathbf{A}}{\partial t}, \quad \mathbf{B}=\nabla \times \mathbf{A} .
$$

The generalized momenta are given by

$$
P_{i}=\frac{\partial \mathcal{L}}{\partial \dot{r}_{i}}
$$

and the Euler-Lagrange equations reads

$$
\frac{\mathrm{d}}{\mathrm{d} t}\left(\frac{\partial \mathcal{L}}{\partial \dot{r}_{i}}\right)=\frac{\mathrm{d} P_{i}}{\mathrm{~d} t}=\frac{\partial \mathcal{L}}{\partial r_{i}}
$$

Thus, using the standard toroidal coordinates $(r, \theta, \zeta)$ introduced in section 2, we get the generalized (canonical) angular momentum

$$
P_{\zeta}=R\left(m v_{\zeta}+e A_{\zeta}\right),
$$

where $A_{\zeta}=\mathbf{A} \cdot \hat{\zeta}$ is the projection of $\mathbf{A}$ in the toroidal direction (i.e. not the covariant $\zeta$ component of $\mathbf{A}$ ), and the toroidal speed is $v_{\zeta}=R \dot{\zeta}$. The evolution equation for $P_{\zeta}$ is given by the Euler-Lagrange equation (A.9). It reads

$$
\frac{\mathrm{d} P_{\zeta}}{\mathrm{d} t}=-e \frac{\partial \phi}{\partial \zeta}+e \dot{\mathbf{r}} \cdot \frac{\partial \mathbf{A}}{\partial \zeta}
$$

We can thus conclude that $P_{\zeta}$ is conserved in axisymmetric devices, where $\frac{\partial \phi}{\partial \zeta}=\frac{\partial \mathbf{A}}{\partial \zeta}=0$. In the presence of the wave field (26) however, axisymmetry is broken, and $P_{\zeta}$ is no longer an invariant. We then have

$$
\mathbf{E}_{1}=-\nabla \phi_{1}-\frac{\partial \mathbf{A}_{1}}{\partial t},
$$

and by imposing the Coulomb gauge condition $\nabla \cdot \mathbf{A}_{0}=$ $\mathbf{k} \cdot \mathbf{A}_{1}=0$, equation (A.11) takes the form

$$
\frac{\mathrm{d} P_{\zeta}}{\mathrm{d} t}=e \frac{k_{\zeta} R}{\omega}\left[\mathbf{v} \cdot \mathbf{E}_{k}+(\omega-\mathbf{k} \cdot \mathbf{v}) \frac{\mathbf{k} \cdot \mathbf{E}_{k}}{k^{2}}\right] \mathrm{e}^{-\mathrm{i} \omega t+\mathrm{i} \mathbf{k} \cdot \mathbf{r}},
$$

where $k_{\zeta}=-\frac{n}{R}$. Note that upon integrating to obtain $P_{\zeta}$ as a function of time, the second term inside the bracket behaves as a total derivative which can be evaluated directly.

\section{References}

[1] Rosenbluth M N 1972 Phys. Rev. Lett. 29408

[2] Timofeev A V 1974 Nucl. Fusion 14165

[3] Zaslavsky G M and Chirikov B V 1972 Sov. Phys.-Usp. 14549

[4] Chirikov B V 1960 J. Nucl. Energy C 1253

[5] Chirikov B V 1979 Phys. Rep. 52263

[6] Smith G R and Kaufman A N 1978 Phys. Fluids 212230

[7] Smith G R 1977 Phys. Rev. Lett. 38970

[8] Smith G R, Byers J A and LoDestro L L 1980 Phys. Fluids 23278

[9] Shimomura Y and Odijama K 1987 Commun. Plasma Phys. X 207

[10] Helander P and Lisak M 1992 Phys. Fluids B 41927

[11] Belikov V S and Kolesnichenko Ya I 1994 Plasma Phys. Control. Fusion 361703

[12] Belikov V S and Kolesnichenko Ya I 1987 Nucl. Fusion 271371

[13] Marchenko V S 1994 Nucl. Fusion 34740

[14] Fukuyama A, Momota H, Itatani R and Takizuka T 1977 Phys. Rev. Lett. 38701

[15] Karney C F F and Bers A 1977 Phys. Rev. Lett. 39550

[16] Karney C F F 1979 Phys. Fluids 211584 Karney C F F 1979 Phys. Fluids 222188

[17] Morozov A I and Solov'ev L S 1996 Reviews of Plasma Physics vol 2 (New York: Consultants Bureau) 